NORDITA-93/13 N,P

UAB-FT-299/92

UG-FT-27/92

\title{
SEMILEPTONIC $\pi$ AND K DECAYS AND THE CHIRAL ANOMALY AT ONE-LOOP
}

\author{
Ll. AMETLLER \\ Departament Física i Enginyeria Nuclear, FIB, UPC, \\ 08028 Barcelona, Spain \\ J. BIJNENS \\ NORDITA, Blegdamsvej 17, \\ DK-2100 Copenhagen Ø, Denmark \\ A. BRAMON \\ Grup de Física Teòrica, Universitat Autònoma de Barcelona, \\ 08193 Bellaterra (Barcelona), Spain \\ and \\ F. CORNET \\ Departamento de Física Teórica y del Cosmos, \\ Universidad de Granada, 18071 Granada, Spain
}

\begin{abstract}
We calculate the $O\left(p^{6}\right)$ corrections to the anomalous form factors appearing in $\pi^{+}$, $K^{+} \rightarrow e^{+} \nu \gamma, \mu^{+} \nu \gamma$ and $K_{l 4}$ decays in Chiral Perturbation Theory. The relevant dimension 6 terms of the lagrangian are evaluated assuming their saturation by the vector meson contribution.
\end{abstract}


Chiral Perturbation Theory (ChPT) is believed to furnish one of the most appropriate and accurate descriptions of the low energy interactions of pseudoscalar mesons. This includes not only their strong interactions, but also the electroweak semileptonic ones, whose dynamics is completely fixed once the corresponding gauge bosons have been introduced through the usual covariant derivative. The perturbative or series expansion in ChPT is made in terms of the particle four-momenta and pseudoscalar masses, which are therefore required to be small compared to a typical symmetry breaking scale assumed to be around $1 \mathrm{GeV}$ [1] [2]. For all these reasons, the semileptonic and radiative weak decays of pions and kaons are expected to be most accurately described in ChPT. Indeed, one has to deal exclusively with well fixed (no free-parameter) Lagrangians and the energies involved belong to the lowest region of the spectrum, with the masses of the two lightest hadrons as an upper bound. Our purpose is to compute and discuss the anomalous form factor of the $\pi, K \rightarrow \gamma l \nu$ and $K_{l 4}$ decays. In this sense, the present note is an extension of the recent work of one of the authors on the non-anomalous sector of $K_{l 4}$ decays, to which we refer for further details [3] (see also Refs. [4, 5, 6).

The octet of pseudoscalar mesons $M=\sum_{a} \pi^{a} \lambda^{a} / \sqrt{2}$ appears in ChPT through the $3 \times 3$ matrix

$$
\Sigma \equiv \exp \frac{2 i}{f} M
$$

where $\mathrm{f}$ can be identified with the pion decay constant $f_{\pi}=f=132 \mathrm{MeV}\left(=f_{K}\right.$, at lowest order). As previously stated, the electroweak gauge fields $A_{\mu}$ and $W_{\mu}$ are introduced via the covariant derivative

$$
\begin{aligned}
& D_{\mu}=\partial_{\mu} \Sigma+i L_{\mu} \Sigma-i \Sigma R_{\mu} \\
& L_{\mu}=e A_{\mu} Q-\frac{g}{\sqrt{2}}\left(\begin{array}{ccc}
0 & \cos \theta W_{\mu}^{+} & \sin \theta W_{\mu}^{+} \\
\cos \theta W_{\mu}^{-} & 0 & 0 \\
\sin \theta W_{\mu}^{-} & 0 & 0
\end{array}\right) ; \quad R_{\mu}=e A_{\mu} Q,
\end{aligned}
$$

where $Q$ is the quark charge matrix in units of $e=g \sin \theta_{W}>0$, and $\theta$ and $\theta_{W}$ stand for the Cabibbo and Weinberg angles. The final lepton pair (for definiteness we will always refer to $l^{+} \nu$ coming a $\left.W^{+}\right)$appears in the leptonic current $l_{\mu}$ when substituting

$$
W_{\mu} \rightarrow \frac{g}{2 \sqrt{2} M_{W}^{2}} l_{\mu} \equiv \frac{g}{2 \sqrt{2} M_{W}^{2}} \bar{u}_{\nu} \gamma_{\mu}\left(1-\gamma_{5}\right) v_{e}
$$

with $G_{F} / \sqrt{2}=g^{2} / 8 M_{W}^{2}$.

The Lagrangian of ChPT has an anomalous and a normal (or non-anomalous) sector. At lowest order ( $p^{2}$ or second order), the latter is fully given by

$$
L_{2}=\frac{1}{8} f^{2} \operatorname{tr}\left[D_{\mu} \Sigma D^{\mu} \Sigma^{\dagger}+\chi \Sigma^{\dagger}+\Sigma \chi^{\dagger}\right]
$$


where the last $\chi$-terms contain the $\mathrm{SU}(3)$-breaking quark mass matrix. The anomalous sector begins at fourth $\left(p^{4}\right)$ order with the Wess-Zumino term $L_{W Z}$ [7] containing pieces (among others) with zero, one and two gauge boson fields. The five pseudoscalar (no-boson) term relevant for our purposes is given by

$$
L_{W Z}^{(0)}=-\frac{2}{5 \pi^{2} f^{5}} \epsilon^{\mu \nu \alpha \beta} \operatorname{tr}\left(M \partial_{\mu} M \partial_{\nu} M \partial_{\alpha} M \partial_{\beta} M\right) .
$$

The terms with one and two gauge bosons have the form

$$
\begin{aligned}
L_{W Z}^{(1)}= & -\frac{1}{16 \pi^{2}} \epsilon^{\mu \nu \alpha \beta} \operatorname{tr}\left(\Sigma \partial_{\mu} \Sigma^{\dagger} \partial_{\nu} \Sigma \partial_{\alpha} \Sigma^{\dagger} L_{\beta}-\Sigma^{\dagger} \partial_{\mu} \Sigma \partial_{\nu} \Sigma^{\dagger} \partial_{\alpha} \Sigma R_{\beta}\right) \\
L_{W Z}^{(2)}= & -\frac{i}{16 \pi^{2}} \epsilon^{\mu \nu \alpha \beta} \operatorname{tr}\left[\partial_{\mu} \Sigma^{\dagger} \partial_{\nu} L_{\alpha} \Sigma R_{\beta}-\partial_{\mu} \Sigma \partial_{\nu} R_{\alpha} \Sigma^{\dagger} L_{\beta}\right. \\
& \left.+\Sigma \partial_{\mu} \Sigma^{\dagger}\left(L_{\nu} \partial_{\alpha} L_{\beta}+\partial_{\nu} L_{\alpha} L_{\beta}\right)\right],
\end{aligned}
$$

where only the terms needed for the processes discussed here are given.

With these Lagrangians one immediately obtains the tree-level amplitude for the anomalous part of our processes. For the radiative $\pi$ and $K$ weak decays one has

$$
\begin{aligned}
& A^{a n}(\pi \rightarrow l \nu \gamma)=\frac{e G_{F} \cos \theta}{\sqrt{2} m_{\pi}} F_{V}^{\pi} \epsilon^{\mu \nu \alpha \beta} l_{\mu} q_{\nu} \varepsilon_{\alpha} k_{\beta} \\
& A^{a n}(K \rightarrow l \nu \gamma)=\frac{e G_{F} \sin \theta}{\sqrt{2} m_{\pi}} F_{V}^{K} \epsilon^{\mu \nu \alpha \beta} l_{\mu} q_{\nu} \varepsilon_{\alpha} k_{\beta},
\end{aligned}
$$

with $F_{V}^{\pi}=m_{\pi} / 4 \pi^{2} f$ and $F_{V}^{K}=m_{K} / 4 \pi^{2} f$. For the different $K_{l 4}$ amplitudes one similarly get:

$$
A^{a n}\left(K \rightarrow \pi^{i} \pi^{j} l \nu\right)=-\sqrt{2} G_{F} \sin \theta \frac{1}{m_{K}^{3}} H^{i j} \epsilon^{\mu \nu \alpha \beta} l_{\mu} q_{\nu} p_{\alpha}^{i} p_{\beta}^{j}
$$

where the indexes $\mathrm{i}, \mathrm{j}=+,-, 0$ refer to the pion charge states and $H^{00}=0, H^{+-}=H^{-0} / \sqrt{2}=$ $-m_{K}^{3} / 2 \pi^{2} f^{3}$. Among these three isospin rotated amplitudes one can verify the relation $A^{-0}=$ $\sqrt{2}\left(A^{+-}-A^{00}\right)$ valid not only at this tree-level but at all orders. In the amplitudes above, $K, p^{i, j}, k$ and $q$ stand for the four-momenta of the decaying pseudoscalar, final pions, photon (with polarization $\varepsilon$ ) and lepton pair, respectively, i.e., $K=q+k$ or $K=p^{i}+p^{j}+q$.

One-loop corrections to the above tree-level amplitudes proceed through diagrams similar to those in Refs. [3, 8], as well as from the usual renormalization of the pseudoscalar wave-functions and decay constants. For the two radiative decays we have found

\footnotetext{
${ }^{1}$ The normalization of the $K_{l 4}$ form factors used here is that of Ref. [3]. Conversion to those of Ref. 5] can be done by comparing the respective lowest order expressions.
} 


$$
\begin{aligned}
& A^{a n}(\pi \rightarrow l \nu \gamma)= \frac{e G_{F} \cos \theta}{4 \sqrt{2} \pi^{2} f_{\pi}} \epsilon^{\mu \nu \alpha \beta} l_{\mu} q_{\nu} \varepsilon_{\alpha} k_{\beta}\left\{1+\frac{1}{16 \pi^{2} f^{2}}\left[\frac{2}{3} \lambda\left(k^{2}+q^{2}\right)\right.\right. \\
&\left.\left.-4 m_{\pi}^{2} \ln \frac{m_{\pi}^{2}}{\mu^{2}}-4 m_{K}^{2} \ln \frac{m_{K}^{2}}{\mu^{2}}+4 I\left(q^{2}, m_{\pi}^{2}, m_{\pi}^{2}\right)+4 I\left(k^{2}, m_{K}^{2}, m_{K}^{2}\right)\right]+C_{\pi}\right\}, \\
& A^{a n}(K \rightarrow \quad l \nu \gamma)=\frac{e G_{F} \sin \theta}{4 \sqrt{2} \pi^{2} f_{K}} \epsilon^{\mu \nu \alpha \beta} l_{\mu} q_{\nu} \varepsilon_{\alpha} k_{\beta}\left\{1+\frac{1}{16 \pi^{2} f^{2}}\left[\frac{5}{3} \lambda\left(m_{K}^{2}-m_{\pi}^{2}\right)\right.\right. \\
&+\frac{2}{3} \lambda\left(k^{2}+q^{2}\right)-\frac{7}{2} m_{\pi}^{2} \ln \frac{m_{\pi}^{2}}{\mu^{2}}-3 m_{K}^{2} \ln \frac{m_{K}^{2}}{\mu^{2}}-\frac{3}{2} m_{\eta}^{2} \ln \frac{m_{\eta}^{2}}{\mu^{2}} \\
&\left.\left.+4 I\left(k^{2}, m_{\pi}^{2}, m_{\pi}^{2}\right)+2 I\left(q^{2}, m_{K}^{2}, m_{\pi}^{2}\right)+2 I\left(q^{2}, m_{K}^{2}, m_{\eta}^{2}\right)\right]+C_{K}\right\} .
\end{aligned}
$$

Similarly, for the various $K_{l 4}$ amplitudes we obtain

$$
\begin{aligned}
& A^{a n}\left(K^{+} \rightarrow \pi^{+} \pi^{-} l^{+} \nu\right)=\frac{G_{F} \sin \theta}{\sqrt{2} \pi^{2} f_{K} f_{\pi}^{2}} \epsilon^{\mu \nu \alpha \beta} l_{\mu} q_{\nu} p_{\alpha}^{+} p_{\beta}^{-} \\
& \left\{1+\frac{1}{16 \pi^{2} f^{2}}\left[\frac{7}{6} \lambda\left(m_{\pi}^{2}-m_{K}^{2}\right)+\frac{1}{3} \lambda\left[\left(p^{+}+p^{-}\right)^{2}+\left(p^{-}+q\right)^{2}+2 q^{2}\right]\right.\right. \\
& -\frac{11}{2} m_{\pi}^{2} \ln \frac{m_{\pi}^{2}}{\mu^{2}}-5 m_{K}^{2} \ln \frac{m_{K}^{2}}{\mu^{2}}-\frac{3}{2} m_{\eta}^{2} \ln \frac{m_{\eta}^{2}}{\mu^{2}} \\
& +2 I\left(\left(p^{+}+p^{-}\right)^{2}, m_{\pi}^{2}, m_{\pi}^{2}\right)+I\left(\left(p^{+}+p^{-}\right)^{2}, m_{K}^{2}, m_{K}^{2}\right)+2 I\left(\left(p^{-}+q\right)^{2}, m_{K}^{2}, m_{\pi}^{2}\right) \\
& \left.\left.+I\left(\left(p^{-}+q\right)^{2}, m_{K}^{2}, m_{\eta}^{2}\right)+3 I\left(q^{2}, m_{K}^{2}, m_{\pi}^{2}\right)+3 I\left(q^{2}, m_{K}^{2}, m_{\eta}^{2}\right)\right]+C_{+-}\right\}, \\
& A^{a n}\left(K^{+} \rightarrow \pi^{0} \pi^{0} l^{+} \nu\right)=\frac{G_{F} \sin \theta}{\sqrt{2} \pi^{2} f_{K} f_{\pi}^{2}} \epsilon^{\mu \nu \alpha \beta} l_{\mu} q_{\nu} p_{\alpha} p_{\beta}^{\prime} \\
& \left\{0+\frac{1}{16 \pi^{2} f^{2}}\left[-\frac{1}{3} \lambda\left[(q+p)^{2}-\left(q+p^{\prime}\right)^{2}\right]\right.\right. \\
& -I\left((q+p)^{2}, m_{K}^{2}, m_{\pi}^{2}\right)-\frac{1}{2} I\left((q+p)^{2}, m_{K}^{2}, m_{\eta}^{2}\right) \\
& \left.\left.+I\left(\left(q+p^{\prime}\right)^{2}, m_{K}^{2}, m_{\pi}^{2}\right)+\frac{1}{2} I\left(\left(q+p^{\prime}\right)^{2}, m_{K}^{2}, m_{\eta}^{2}\right)\right]+C_{00}\right\} .
\end{aligned}
$$


The $A^{00}$ amplitude is the even part of the $A^{+-}$amplitude under the interchange $p^{i} \leftrightarrow p^{j}$ as required by isospin. The $A^{-0}$ amplitudes are the odd part of $A^{+-}$or can be determined by the relation discussed above.

All the four one-loop amplitudes above have been expressed in a similar form. The first term inside the brackets (vanishing in the last amplitude) is essentially the tree-level term quoted in eqs.(7), (8). Notice however that the latter eqs. always contain the unbroken decay constant $\mathrm{f}$, whereas in the one-loop corrected versions one has the corresponding $f_{\pi}$ or $f_{K}$ decay constant. Next, one has two types of divergent terms proportional to $\lambda=1 / \epsilon+1+\ln 4 \pi-\gamma_{E}$ coming from our dimensional regularization scheme, which also introduces the subtraction mass $\mu$. These terms will be discussed later on together with the contributions from the higher order effective Lagrangian, the $C_{i}$. Finally, one has the so called chiral logs incorporating explicit logarithmic terms plus the function $I$ to which we now turn.

The defining equation for the function $I$ and its relation to the simpler functions $A$ and $B$ is given by

$$
\begin{aligned}
& I\left(k^{2}, m_{1}^{2}, m_{2}^{2}\right) \equiv \\
& \int_{0}^{1} d x\left[m_{1}^{2}-\left(m_{1}^{2}-m_{2}^{2}\right) x-x(1-x) k^{2}\right] \ln \frac{\left[m_{1}^{2}-\left(m_{1}^{2}-m_{2}^{2}\right) x-x(1-x) k^{2}\right]}{\mu^{2}} \\
& =16 \pi^{2}\left[\frac{m_{1}^{2}-m_{2}^{2}+k^{2}}{6 k^{2}} i A\left(m_{1}^{2}\right)+\frac{-m_{1}^{2}+m_{2}^{2}+k^{2}}{6 k^{2}} i A\left(m_{2}^{2}\right)\right. \\
& \left.\quad-\frac{\left(m_{1}^{2}-m_{2}^{2}-k^{2}\right)^{2}-4 k^{2} m_{2}^{2}}{6 k^{2}} i B\left(k^{2}, m_{1}^{2}, m_{2}^{2}\right)\right]-\frac{1}{3}\left(m_{1}^{2}+m_{2}^{2}\right)+\frac{1}{9} k^{2},
\end{aligned}
$$

where

$$
i A\left(m^{2}\right)=\frac{m^{2}}{16 \pi^{2}} \ln \frac{m^{2}}{\mu^{2}}
$$

and

$$
i B\left(k^{2}, m_{1}^{2}, m_{2}^{2}\right)=\frac{-1}{16 \pi^{2}}\left\{1-\frac{1}{2} \log \frac{m_{1}^{2} m_{2}^{2}}{\mu^{4}}+\frac{m_{2}^{2}-m_{1}^{2}}{2 k^{2}} \log \frac{m_{1}^{2}}{m_{2}^{2}}-\frac{1}{k^{2}} u_{+} u_{-} \log \frac{u_{+}+u_{-}}{u_{+}-u_{-}}\right\},
$$

with $u_{ \pm}=\sqrt{k^{2}-\left(m_{1} \pm m_{2}\right)^{2}}$, coincide with the first two equations of Appendix A in ref. [3] with the opposite signs. In particular, for $m_{1}=m_{2}$ and small values of $k^{2}$ one has

$$
I\left(k^{2}, m^{2}, m^{2}\right)=m^{2} \ln \frac{m^{2}}{\mu^{2}}-\frac{k^{2}}{6}\left(1+\ln \frac{m^{2}}{\mu^{2}}\right)+\mathcal{O}\left(k^{4}\right)
$$

This expansion allows for a clear check of our expressions: making all the various squared fourmomenta in the $I$-integrals tend to zero and using the last expansion implies the vanishing of 
all the chiral logs, in agreement with the well-known non-renormalization of the anomaly in the soft limit.

As previously mentioned, there are two types of divergent $\lambda$-terms. Some are proportional to the $\mathrm{SU}(3)$-breaking mass-difference $m_{K}^{2}-m_{\pi}^{2}$ and have been derived using the Gell-Mann-Okubo relation $\left(3 m_{8}^{2}=4 m_{K}^{2}-m_{\pi}^{2}\right)$ to eliminate the $\eta$-mass. The other type of divergent terms contain the various combinations of squared four-momenta in the final state. Again, both types of terms vanish in the soft limit for masses and four-momenta. Also, and this is another test of our calculation, these two types of terms could be expected on the basis of the analysis made in ref. [8]. In this paper, all the divergent terms appearing at the one loop level in the anomalous sector were identified and classified in two groups: the SU(3)breaking terms proportional to the M-meson squared mass differences and terms containing combinations of squared four-momenta. It is easy to identify in each group of this general expression each one of the divergent terms obtained in eqs. (9-12). Obviously, the presence of these divergent terms requires the introduction of the corresponding counterterms in the anomalous part of the Lagrangian at order $p^{6}$. Examples of these counterterms were already given in refs. [9], [10]. Their infinite part cancels the $\lambda$-terms in eqs. (9 12), which are simply substituted by the remaining finite part of the counterterms, $C_{i}^{r}$. The value of these finite contributions from the counterterms to our processes is not fixed in ChPT. It has to be deduced from data fitting (as was done in ref. [1, 5] for the counterterms of the non-anomalous Lagrangian at order $p^{4}$ ) or, alternatively, from the hypothesis of resonance saturation of the counterterms.

This hypothesis of resonance saturation was already proposed by Gasser and Leutwyler in their original papers on ChPT [何] and has been fully exploited and confirmed by Ecker et al. [11] and other authors. In our case involving photons, lepton currents and pseudoscalar pairs, one expects the vector-mesons to play the essential role. These are easily incorporated in the chiral Lagrangian as "massive" Yang-Mills fields [12] or in the so called "hidden symmetry" scheme of Bando et al. [13]. For our processes, all the relevant dynamics is generated by the vector-vector-pseudoscalar (VVM) coupling discussed in refs. [8] and [10], where details can be found. The contribution of vector-mesons to the first type of ( $\mathrm{SU}(3)$-breaking) counterterms is found to vanish in all our amplitudes. By contrast, it is found to give a definite finite contribution to the other type (four-momenta dependent) counterterms. For the two radiative weak decays, vector-meson dominance of the counterterms implies that in eqs. (9) and (10) one has

$$
C_{\pi}^{r}=C_{K}^{r}=\frac{k^{2}}{M_{V}^{2}}+\frac{q^{2}}{M_{V}^{2}}+\ldots
$$

where the dots stand for contributions of heavier resonances than the vector mesons. As expected, this $k^{2}$ and $q^{2}$ dependence coincides with the one obtained in ref. [9] when dealing 
with the purely anomalous $\pi^{0}, \eta$ couplings to two off-mass-shell photons. For $K_{l 4}$ decays, the vector meson contributions give in eq. (11)

$$
C_{+-}^{r}=\frac{3}{2} \frac{q^{2}}{M_{V}^{2}}+\frac{3}{4} \frac{\left(p^{+}+p^{-}\right)^{2}}{M_{V}^{2}}+\frac{3}{4} \frac{\left(q+p^{-}\right)^{2}}{M_{V}^{2}}+\ldots
$$

where the dots again refer to contributions from heavier resonances. These $M_{V}^{2}$ dependent terms are the analogues of those found in ref. [10] when studying the $\eta \rightarrow \pi^{+} \pi^{-} \gamma$ decays. Similarly, in eq. (12) we obtain $C_{00}^{r}=3(q+p)^{2} / 8 M_{V}^{2}-3\left(q+p^{\prime}\right)^{2} / 8 M_{V}^{2}+\ldots$

Having calculated the vector-meson contribution to our one-loop amplitudes we are able to present our predictions. These are summarized in Figs. 1 and 2 for the decays $\pi^{+} \rightarrow e^{+} \nu \gamma$ and $K^{+} \rightarrow e^{+} \nu \gamma$, respectively. The quantities plotted, $F^{P}$, are the respective form factors normalized to 1 for vanishing masses and four-momenta:

$$
F^{P}=\frac{4 \pi^{2} f_{P}}{m_{P}} F_{V}^{P}
$$

These form factors are also valid for the decays with muons in the final state. These are, however, suppressed by phase space compared to the decays involving electrons. In both cases the $O\left(p^{6}\right)$ corrections introduce a dependence of the form factors with the $e^{+} \nu$ and photon invariant masses. The corrections obtained for the $\pi$ radiative decay are very small (smaller than 5\%) all over the allowed kinematical range (Fig. 1). The case for $K^{+} \rightarrow e^{+} \nu \gamma$ is more interesting because the corrections are much larger (Fig. 2). They can become as large as $\sim 50 \%$ at the edge of the allowed kinematical range. There the number of events is, however, very small. In any case, in the kinematical regions where the number of events is larger the corrections can be as large as $10-20 \%$. Finally, in Fig. 3 we present our results for the $\mathrm{H}^{+-}$form factor as a function of the $\left(\pi^{+} \pi^{-}\right)$invariant mass, and we compare them to the experimental data [14]. We have evaluated the form factor taking a lepton invariant mass of $100 \mathrm{MeV}$ and a $\cos \theta_{\pi}$ of 0.2 . We keep these values fixed because this was how the experiment was analyzed and these values represent the peak of the observed contributions. The lowest order prediction with $f_{K}=f_{\pi}, H^{+-}=-2.67$, is given by the dotted line. The predicted value of $H^{+-}$increases when one takes the lowest order expression with $f_{K}=1.2 f_{\pi}$ (dashed line), as is sometimes done in current algebra. However, when the effects of the loop corrections and the higher order terms in the lagrangian are consistently taken into account, the previous increase is almost canceled. So, the total prediction turns out to be very similar to the lowest order one with $f_{K}=f_{\pi}$.

The question of the saturation of the counterterms only by vector mesons deserves some comments. It is certainly very reasonable for the second (four-momenta dependent) type of counterterms, but the vanishing contribution generated by this saturation to the 
other type of counterterms looks more suspicious. To generate a non-vanishing contribution one should invoke the presence of scalar resonances with known couplings to $M W \gamma$ states. Experimentally nothing is known about this type of couplings; even the nature and properties of the scalar mesons are highly controversial. There are two indications that the effects of the scalar mesons are rather small. In the non-anomalous sector (see [11]) their couplings tend to be smaller than those of the vector mesons and, in addition, their contributions are suppressed by an extra factor of $M_{S}^{2} / M_{V}^{2} \approx 0.6$. The other indication suggesting that such scalar mesons contributions are small follows from our previous work on the $\pi^{0}, \eta$ coupling to virtual photons [9], where similar contributions should be present. The good agreement obtained between the data and the predictions from ChPT without scalar meson contributions can be interpreted as an indication of the smallness of the latter.

In summary, we have discussed de $O\left(p^{6}\right)$ corrections to the anomalous form factors in $\pi^{+}, K^{+} \rightarrow e^{+} \nu \gamma$ and $K_{l 4}$ decays. These include the corrections from loop diagrams and the ones from higher dimension terms in the lagrangian. The former can be exactly calculated in terms of the known parameters of the lagrangian. In order to estimate the latter we have assumed that they are saturated by the vector meson contribution. In $\pi^{+} \rightarrow e^{+} \nu \gamma$ and $K^{+} \rightarrow$ $\pi^{+} \pi^{-} e^{+} \nu$ the corrections obtained are very small. The case of $K_{l 4}$ is particularly interesting because there is a cancellation between the effects of $S U(3)$-breaking correction in the meson decay constants and the contribution from the loop diagrams, higher dimension terms and wave function renormalization. Unfortunately, the errors in the available experimental data on this decay are too large to extract any conclusion. Finally, the corrections obtained for the decay $K^{+} \rightarrow e^{+} \nu \gamma$ are larger. In all the cases the main contribution to the corrections is given by the $O\left(p^{6}\right)$ tree level terms.

\section{References}

[1] J. Gasser and H. Leutwyler, Nucl.Phys.B250 (1985) 465.

[2] H. Leutwyler, Proceedings of the XXVI International Conference on High Energy Physics, Dallas 1992.

[3] J. Bijnens, Nucl. Phys. B337 (1990) 635.

[4] C. Riggenbach et al., Phys. Rev. D43 (1991) 127.

[5] J. Bijnens, G. Ecker and J. Gasser, Semileptonic kaon decays in Chiral Perturbation Theory, in "DA $\Phi$ E Physics Handbook," eds. L. Maiani, G. Pancheri and N. Paver, INFN-Frascati, 1992. 
[6] J. Bijnens, G. Ecker and J. Gasser, Radiative semileptonic kaon decays, preprint CERNTH 6625/92, BUTP-92/38.

[7] J. Wess and B. Zumino,Phys. Lett. B37 (1971) 95,

E. Witten, Nucl. Phys. B233 (1983) 422.

[8] J. Bijnens, A. Bramon and F. Cornet, Z. Phys. C 46 (1990) 599; see also D. Issler, SLAC report SLAC-PUB-4943 (1989).

[9] J. Bijnens, A. Bramon and F. Cornet, Phys. Rev. Lett. 61 (1988) 1453; J. Donoghue and D. Wyler, Nucl. Phys. B316 (1989) 289

[10] J. Bijnens, A. Bramon and F. Cornet, Phys. Lett. B237 (1990) 488; Ll. Ametller et al., in Proceedings of the Daphne Workshop; ed. G. Pancheri, INFN; Frascati 1991.

[11] G. Ecker, J. Gasser, A. Pich and E. de Rafael, Nucl. Phys. B321 (1989) 311. See also J. F. Donoghue, C. Ramirez and G. Valencia, Phys. Rev. D39 (1989) 1947.

[12] U.-G. Meissner, Phys. Rep. 161 (1988) 213.

[13] M. Bando et al., Phys. Rep. 164 (1988) 217.

[14] L. Rosselet et al., Phys. Rev. D15 (1977) 574. 


\section{Figure Captions}

Fig. 1: $F^{\pi}$ form factor at $O\left(p^{6}\right)$ (normalized to one for vanishing masses and four momenta) for $\pi^{+} \rightarrow l^{+} \nu \gamma$ as a function of the photon squared invariant mass, $k^{2}$, and the $l^{+} \nu$ squared invariant mass, $q^{2}$.

Fig. 2: $F^{K}$ form factor at $O\left(p^{6}\right)$ (normalized to one for vanishing masses and four momenta) for $K^{+} \rightarrow l^{+} \nu \gamma$ as a function of the photon squared invariant mass, $k^{2}$, and the $l^{+} \nu$ squared invariant mass, $q^{2}$.

Fig. 3: $H^{+-}$form factor at $O\left(p^{6}\right)$ for $K^{+} \rightarrow \pi^{+} \pi^{-} l^{+} \nu$ as a function of the $\pi^{+} \pi^{-}$ invariant mass $E_{\pi \pi}$. 